\title{
VENOMENON - A multi-layered visual experience
}

\author{
Elke E. Reinhuber \\ School of Art, Design and Media, \\ Nanyang Technological University \\ 81 Nanyang Drive \#03-09 \\ 637458 Singapore \\ elke@ntu.edu.sg
}

\section{INTRODUCTION}

Based on specifically produced S3D video footage, the artistic research explores different ways of presentation to engage an active and mobile spectator in the media art project VENOMENON.

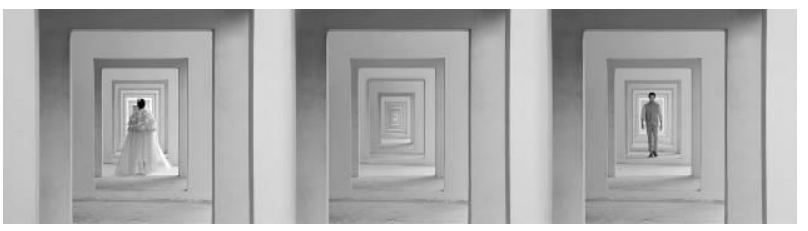

Figure 1: VENOMENON. $360^{\circ}$ version for Oculus Rift or Google Cardboard (Filmstill), Elke Reinhuber, 2015

\section{THE PROJECT}

With the current popularity of $360^{\circ}$ movies for immersive VR environments and non-linear media such as video games, filmmakers and media artists are in demand to reconsider narrative structures. The artistic research VENOMENON investigates if an audience might be conveyed to the situation of the protagonist by the particular form of presentation in a movie short.

Fairy-tales, fables and sagas belong to the foundations of our narrative culture - in current movies, the epic television series of today or contemporary video games, vestiges of antique mythologies are always recognisable. Since the narrative concepts of ancient myths are still valid nowadays, we can explore the topics as foundation for story-telling strategies. Based on the various readings of the ancient Greek legend of Orpheus and Eurydice, the plot offers a wide range of possible interpretations. The male hero lost his wife and seeks to rescue her from the underworld. While nearly having succeeded with this impossible task, he turned around to look back at her despite the impediment not to do so - and lost his wife in the classic tale forever. This physical act of turning one's head is transferred to the viewer by presenting different content on two opposing screens.

In reference to Virgil, Ovid, Plato and later Gluck, Offenbach, Cocteau or Marcel Camus all interpretations of the love-story are possible in the imagination of the audience.

In order to engage the audience accordingly, I examine different settings as described hereafter:

\subsection{S3D video installation on opposing screens}

This method seems to provide in my eyes the ideal form of presentation, a dual-channel installation with two opposing S3D projections, the audience positioned in the centre between both screens, seeing either of them without turning the head and accordingly missing the visuals on the other screen. The application of S3D engages in the sculptural architecture on the screens. Compared to a presentation form with an individual VR headset, which facilitates the presentation of $3 \mathrm{D}$ media, the audience enjoys a collective experience and may physically engage with the simulated depth of the screened spaces.

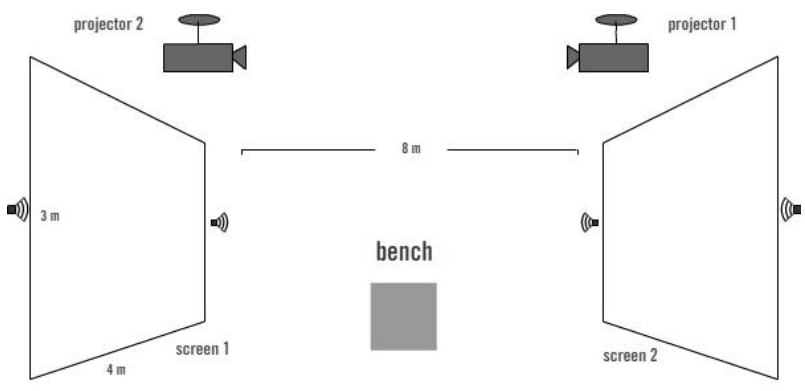

Figure 2: Set-up for VENOMENON as 2-channel $S 3 D$ installation 


\subsection{Spherical projection for $360^{\circ}$ immersive environment}

A second version displays Orpheus' desperate search for his lost wife in the underworld in a $360^{\circ}$ panoramic view. Accessible with a portable virtual reality (VR) device, such as Oculus Rift or Google Cardboard, the spectator immerses oneself in a labyrinth of hallways in which the protagonist follows a sometimes-appearing vision of the female protagonist. While trying to follow the movement of the actors, they keep vanishing and encourage the viewer to turn the head around. The S3D video footage was applied to a spherical mapping, as required for VR devices. In addition, spatial audio was implemented in Unity and responds to the movement of the head, similar to the experience of special audio in a $360^{\circ}$ environment.

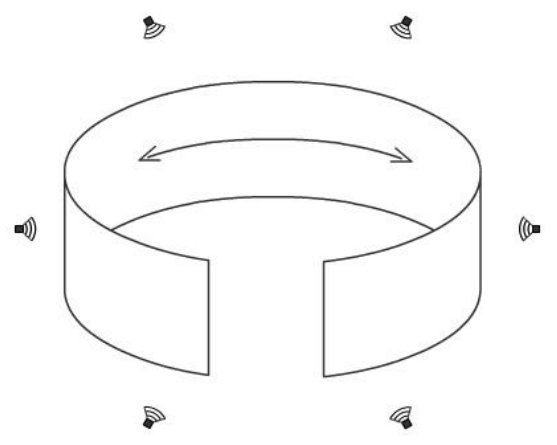

Figure 3: Set-up for VENOMENON (VR version for $360^{\circ}$ environments, but also Oculus Rift or Google Cardboard)

\subsection{Single channel video with multiple layers}

All the above mentioned set up arrangements provide access to more than one linear narrative, either with overlays, multiple screens or a spherical projection which exceeds our peripheral vision. In reference to all the different versions of the tale itself, the material leaves freedom for subjective interpretations in regard to the relation of the protagonists. Hence, a single channel version deals with the ambiguity via overlays, which are in some situations contradictory, in others redundant. In this manner, the audience can develop similar counterfactual thoughts as they encounter while turning their heads in the multi screen set up.

The video piece, in any of these above-mentioned settings, is ideally presented with a series of lenticular prints, displaying the ambiguity of the characters with several alternating images at once on wall-mounted pictures, furthermore inducing counterfactual thoughts.

\section{CONSIDERATIONS}

Based on Sigmund Freud's theory of the unconscious, even single screen movies trigger different associations in our mind. Therefore, I consider it essential to provide sufficient freedom for subjective interpretations, created by memories and emotions. In addition, I am responding with the multi-layered installation to the inflationary 'captureall' approach of our everyday life.

Not only in the range of our perception does this sensory overload happen, but far beyond. In VENOMENON, the spectator is immersed in sculptural impressions, surrounded by a spatial audio composition. With visual and acoustic stimulation from different directions a supplementary layer of scientific imaging can be accessed and as well assessed. The insight into the physical conditions of the protagonists on film becomes visible through this supplementary layer. Visualisations, as used in medicine, give a detailed view, although the spectator might not be able to read them without additional knowledge, but will recognise the visual vernacular.

According to the tale, as Orpheus has been the first human being who visited the underworld and returned alive to the here and now, particular attention is drawn to the display of life-supporting imaging technologies, which merely provide a concept of the corporeal, but never on a mental or psychological level.

But soon, more and more of these technologies will become commonplace and used presumably in the most trivial manner. With scientific imaging, it is possible to capture a wide range of details, usually invisible for the human eye. Though, they cannot reveal anything about emotions. Nevertheless we actually have to value and come to terms with these images, since they surround us already.

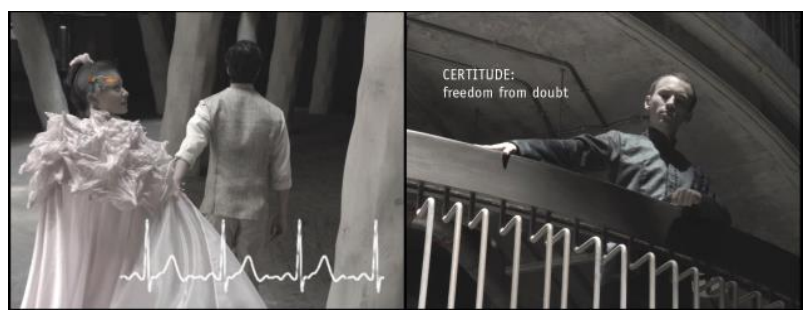

Figure 4: 2D still Venomenon - two opposing screens with various overlays: The Rescue from the Underworld

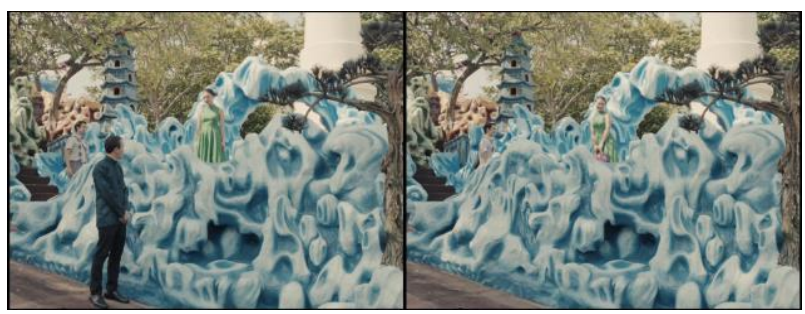

Figure 5: 2D still Venomenon - two opposing screens with different versions: Flashback Scene in Haw Par Villa 